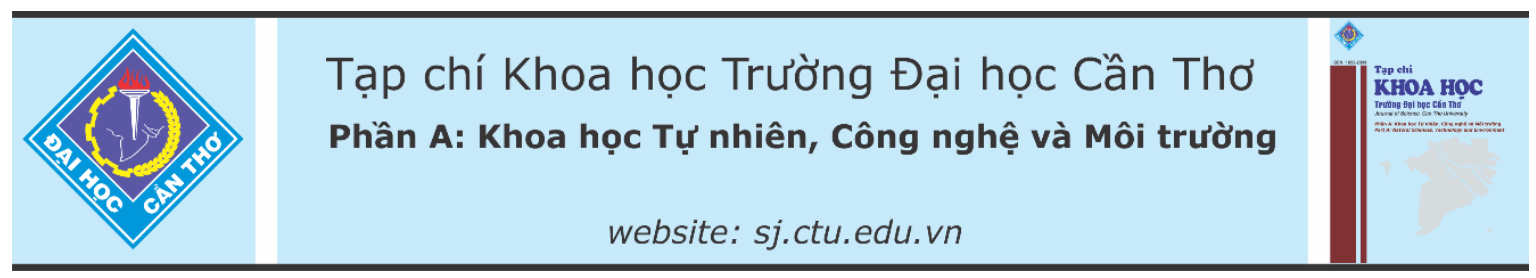

DOI:10.22144/ctu.jvn.2021.004

\title{
LUẠT SỐ LỚN CHO QUÁ TRÌNH KHUẾCH TÁN TRONG KHÔNG GIAN MỘT CHIỀU
}

Lâm Hoàng Chương ${ }^{*}$, Trần Phước Lộc ${ }^{1}$, La Mỹ Kim² và Trần Thị Thiện ${ }^{3}$

${ }^{1}$ Khoa Khoa hoc Tự nhiên, Truờng Đại hoc Cần Tho

${ }^{2}$ Lớp Toán úng dung khóa 43, Truờng Đại hoc Cần Tho

${ }^{3}$ Lớp Toán úng dụng khóa 42, Trưòng Đại học Cần Tho

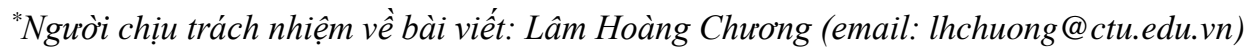

\section{Thông tin chung:}

Ngày nhận bài: 13/08/2020

Ngày nhận bài sưa: 01/10/2020

Ngày duyệt đăng: 27/03/2021

\section{Title:}

Law of large number for diffusion process in one dimension

\section{Tù khóa:}

Luật số lớn, phưong pháp moment, quá trình khuếch tán

\section{Keywords:}

Diffusion process, law of large number, method of moment

\begin{abstract}
The aim of this paper is to study the model of diffusion process in one dimension. The method of moments is used, as in Depauw and Derrien(2009) and Lam (2014) to prove that this process converges in probability to a constant (Theorem 1.1). More precisely, with $L$ be the corresponding infinitesimal generator of the previous process and a given function $f$, we solve the Poisson's equation $L g=f$ and then treat the limits of its solutions, the law of large number is instantly given by the convergence of the moment.
\end{abstract}

\section{TÓM TẮT}

Mục tiêu chính của bài báo là nghiên cưu mô hình quá trình khuếch tán trong một chiều. Phurong pháp moment được sử dụng nhu trong các bài báo Depauw and Derrien (2009) và Lam (2014) để chưng minh sụ họi tụ theo xác suất đến một hằng số của quá trình đang xét (Định lý 1.1). Chi tiết hơn, với L là toán tử cực vi của quá trình đã cho và hàm $f$ cho truớc, bằng cách giải phuơng trình Poisson $L g=f$ rồi sau đó tìm giới hạn liên quan đến nghiệm của nó, một dạng của luật số lớn sẽ được cho bởi sự hộ tụ của các moment.

\section{GIỚI THIỆU}

Xét một quá trình khuếch tán $\left(X_{t}\right)_{t \geq 0}$ với điều kiện ban đầu $X_{0}=0$ và toán tử cực vi $L$ được xác định bởi

$$
\begin{aligned}
L f(k)=\frac{2 \lambda b(x)}{2 a(x)} b^{\prime}(x) & f^{\prime}(x) \\
& +\frac{b(x)}{2 a(x)} f^{\prime \prime}(x)
\end{aligned}
$$

trong đó

$$
\begin{aligned}
& a, b: \mathbb{R} \rightarrow(0 ;+\infty) \text { liên tục } \\
& b^{\prime}: \mathbb{R} \rightarrow \mathbb{R} \text { liên tục } \\
& \lambda: \text { hằng số }
\end{aligned}
$$
$\mathbb{R}$.

và $f$ là hàm đo được trên không gian trạng thái

Quá trình khuếch tán trên thỏa mãn phương trình vi phân ngẫu nhiên

$$
d X_{t}=\sigma\left(X_{t}\right) d B_{t}+\mu\left(X_{t}\right) d t
$$
số

trong đó $\left(B_{t}\right)_{t \geq 0}$ là chuyển động Brown, các hệ

$$
\sigma^{2}(x)=b(x) / a(x) \quad \text { và } \quad \mu(x)=[2 \lambda b(x)+
$$
$\left.b^{\prime}(x)\right] / 2 a(x)$.

Mục tiêu của bài báo là nghiên cứu phân phối giới hạn của $X_{t}$ khi $t \rightarrow+\infty$. 
Mô hình quá trình khuếch tán là một quá trình ngẫu nhiên có nhiều ứng dụng trong thực tế. Đặc biệt trong vật lý động lực học, nó là sự "di chuyển" ngẫu nhiên của một chất điểm trên dây dẫn đồng chất.

Trường hợp $\lambda=0$ thì một dạng của định lý giới hạn trung tâm đã được (Lâm Hoàng Chương và ctv., 2019) chỉ ra, đó là nếu $\lim _{x \rightarrow \infty} a(x)=\alpha>0$, $\lim _{x \rightarrow \infty} b(x)=\beta<+\infty$ thì

$$
\frac{X_{t}}{\sqrt{t}} \stackrel{t \rightarrow+\infty}{\longrightarrow} \mathcal{N}(0 ; \beta / \alpha)
$$

theo phân phối.

Trong phạm vi bài báo này, mô hình của một quá trình khuếch tán $\left(X_{t}\right)_{t \geq 0}$ được xét trên không gian trạng thái $\mathbb{R}$ có điều kiện ban đầu $X_{0}=0$ và hằng số $\lambda>0$. Khi đó, quá trình đã cho sẽ hội tụ theo xác suất đến giá trị phụ thuộc vào $\lambda$ khi thời gian $t$ đủ lớn. Định lý đó được phát biểu như sau:

Định lý 1.1 Giả sử $\lim _{x \rightarrow \infty} a(x)=\alpha, \lim _{x \rightarrow \infty} b(x)=$ $\beta$ và $0<\alpha, \beta<+\infty$ thì $\frac{X_{t}}{t} \stackrel{t \rightarrow+\infty}{\longrightarrow} \frac{\lambda \beta}{\alpha}$

theo xác suất.

Vấn đề này cũng đã được đề cập trong bài báo (Papanicolaou and Varadhan, 1982) trong trường hợp nhiều chiều được chứng minh thông qua phân tích Martingale và với điều kiện các hàm $a(x)$ và $b(x)$ bị chặn nghiêm ngặt, tức là tồn tại các hàng số $C$ và $D$ sao cho $0<C<a(x), b(x)<D<\infty$. Trong bài báo này chỉ cần điều kiện ít hơn cho các hàm trên là $a(x)>0$ và $b(x)<\infty$. Hơn nữa, việc chứng minh Định lý 1.1 được tiến hành thông qua việc sử dụng phương pháp moment.

\section{PHƯƠNG PHÁP NGHIÊN CÚU}

Phương pháp moment lần đầu được giới thiệu bởi Pafnuty Chebyshev trong việc nghiên cứu phân phối giới hạn của dãy các biến ngẫu nhiên. Điểm đặc biệt trong phương pháp này là việc tìm giới hạn của moment bậc $\ell=1,2, \ldots$ và chứng minh sự hội tụ của nó đến moment cùng bậc của biến ngẫu nhiên giới hạn. Để tìm hiểu sâu hơn về phương pháp này bạn đọc có thể tham khảo thêm ở tài liệu (Billingsley, 1995). Bổ đề 2.1 dưới đây là một hệ quả của định lý 30.2 trong tài liệu trên.

Bổ đề 2.1 Cho $\left(Z_{t}\right)_{t \geq 0}$ là một quá trình ngẫu nhiên. Nếu các giới hạn $\lim _{t \rightarrow+\infty} \mathbb{E}\left\{Z_{t}^{\ell}\right\}=A^{\ell}$ với mọi $\ell=1,2$ được thỏa thì $Z_{t}$ hội tụ đến $A$ theo xác suất khi $t \rightarrow+\infty$.

Chứng minh. Áp dụng bất đẳng thức Chebyshev, với mọi $\varepsilon>0$, khi đó

$$
\begin{aligned}
& P\left\{\left|Z_{t}-A\right|>\varepsilon\right\}=P\left\{\left(Z_{t}-A\right)^{2}>\varepsilon^{2}\right\} \\
& \leq \frac{\mathbb{E}\left\{\left(Z_{t}-A\right)^{2}\right\}}{\varepsilon^{2}} \\
& =\frac{\mathbb{E}\left\{Z_{t}^{2}-2 A Z_{t}+A^{2}\right\}}{\varepsilon^{2}} \\
& =\frac{\mathbb{E}\left\{Z_{t}^{2}\right\}-2 A \mathbb{E}\left\{Z_{t}\right\}+A^{2}}{\varepsilon^{2}} \\
& \stackrel{t \rightarrow \infty}{\longrightarrow} 0 \text {. }
\end{aligned}
$$

Trong phần tiếp theo Bổ đề 2.1 sẽ được áp dụng để chứng minh Định lý 1.1

$$
Z_{t}=X_{t} / t \text { và } A=\frac{\lambda \beta}{\alpha} \text {. }
$$

Ngoài ra một số bổ đề liên quan đến giải tích sau đây cũng rất hữu ích trong việc xác định giới hạn của các hàm số:

Bổ đề 2.2 (Phương trình Poisson) Cho trước hàm số $\psi: \mathbb{R} \rightarrow \mathbb{R}$ thì tồn tại hàm $\phi: \mathbb{R} \rightarrow \mathbb{R}$ sao cho

$$
\left\{\begin{array}{l}
L \phi \equiv \psi \\
\phi(0)=0
\end{array}\right.
$$

Bổ đề 2.3 Cho $f(x), g(x)$ là hàm dương, liên tục và $n \in \mathbb{N}$. Giả sử

$$
\lim _{x \rightarrow+\infty} \frac{1}{x} \int_{0}^{x} f(t) d t=\bar{u}, \quad \lim _{x \rightarrow+\infty} g(x)=\bar{v} .
$$

Nếu $\bar{u}$ và $\bar{v}$ hữu hạn thì

$$
\lim _{x \rightarrow+\infty} \frac{1}{x^{n+1}} \int_{0}^{x} t^{n} f(t) g(t) d t=\frac{\bar{u} \bar{v}}{n+1} .
$$

Các bổ đề 2.2 và 2.3 đã được chứng minh trong (Chương và ctv, 2019).

\section{KẾT QUẢ THỰC HIỆN}

Trong phần này ta sẽ chứng minh định lý 2.1 thông qua bổ đề 2.1 . Khi đó ta cần tìm giới hạn của moment bậc 1 và bậc 2 của dãy biến ngẫu nhiên $X_{t} / t$ khi $t$ tiến ra vô cùng. Ý tưởng chủ đạo trong phần chứng minh này là giải phương trình Poisson tương ứng với toán tử cực vi $L$ để tìm nghiệm của nó. Sau đó sử dụng tích phân Ito và một số ước lượng kỳ vọng của biến ngẫu nhiên để đưa đến kết quả mong muốn. 
Mệnh đề 3.1 Với quá trình khuếch tán đã cho

$$
\lim _{t \rightarrow+\infty} \mathbb{E}\left\{\frac{X_{t}}{t}\right\}=\frac{\lambda \beta}{\alpha} \text {. }
$$

Chứng minh. Xét hàm số $f_{1}$, xác định trên $\mathbb{R}$, sao cho

$$
\left\{\begin{array}{l}
L f_{1}(x)=1 \\
f_{1}(0)=0
\end{array} .\right.
$$

Áp dụng Bổ đề 2.2 thì nghiệm của phương trình đã cho là

$$
=\left\{\begin{array}{cc}
\int_{0}^{f_{1}(x)} \frac{1}{e^{2 \lambda v} b(v)} \int_{-\infty}^{v} 2 e^{2 \lambda u} a(u) d u d v, & x \geq 0 \\
-\int_{x}^{0} \frac{1}{e^{2 \lambda v} b(v)} \int_{-\infty}^{v} 2 e^{2 \lambda u} a(u) d u d v, & x<0
\end{array} .\right.
$$

Khi đó giới hạn sau luôn thỏa

$$
\lim _{x \rightarrow \infty} \frac{f_{1}(x)}{x}=\frac{\alpha}{\lambda \beta},
$$

và hơn nữa

$$
\mathbb{E}\left\{f_{1}\left(X_{t}\right)\right\}=t, \quad \forall t \geq 0(3.2)
$$

với mỗi $k \geq 1$.

Chứng minh (3.1). Xét trường hợp $x \geq 0$. Đổi biến $t=v-u$, ta được

$$
\begin{aligned}
f_{1}(x) & =\int_{0}^{x} \frac{1}{e^{2 \lambda v} b(v)} \int_{0}^{+\infty} 2 e^{2 \lambda(v-t)} a(v-t) d t d v \\
& =\int_{0}^{x} \frac{1}{b(v)} \int_{0}^{+\infty} 2 e^{-2 \lambda t} a(v-t) d t d v .
\end{aligned}
$$

Đặt hàm số

$$
h(v)=\int_{0}^{+\infty} 2 e^{-2 \lambda t} a(v-t) d t
$$

và kết hợp với giả thiết $\lim _{x \rightarrow \infty} a(x)=\alpha$ ta được

$$
\begin{array}{r}
\lim _{v \rightarrow \infty} h(v)=\int_{0}^{+\infty} 2 e^{-2 \lambda t} \lim _{v \rightarrow \infty} a(v-t) d t \\
=\alpha \int_{0}^{+\infty} 2 e^{-2 \lambda t} d t=\frac{\alpha}{\lambda}
\end{array}
$$

theo định lý hội tụ bị chặn. Từ đó dẫn đến

$$
\lim _{x \rightarrow \infty} \frac{f_{1}(x)}{x}=\lim _{x \rightarrow \infty} \frac{1}{x} \int_{0}^{x} \frac{h(v)}{b(v)} d v=\frac{\alpha}{\lambda \beta}
$$

theo bổ đề 2.3 kết hợp với giả thiết $\lim _{x \rightarrow \infty} b(x)=$ $\beta$. Ta đặt giới hạn trên là $T(\lambda)=\alpha / \lambda \beta$.

Trường hợp $x<0$, tương tự như trên. Như vậy (3.1) đã được chứng minh.

Chứng minh (3.2). Vì $f_{1} \in C^{2}$ và quá trình $X_{t}$ thỏa mãn phương trình vi phân ngẫu nhiên (1.2) nên theo công thức Ito thì

$$
\begin{aligned}
d f_{1}\left(X_{t}\right)=f_{1}^{\prime}\left(X_{t}\right) & \sigma\left(X_{t}\right) d B_{t} \\
& +\left[f_{1}^{\prime}\left(X_{t}\right) \mu\left(X_{t}\right)\right. \\
& \left.+\frac{1}{2} f_{1}^{\prime \prime}\left(X_{t}\right) \sigma^{2}\left(X_{t}\right)\right] d t \\
=f_{1}^{\prime}\left(X_{t}\right) & \sigma\left(X_{t}\right) d B_{t}+d t .
\end{aligned}
$$

Từ đó

$$
f_{1}\left(X_{t}\right)=f_{1}\left(X_{0}\right)+\int_{0}^{t} f_{1}^{\prime}\left(X_{t}\right) \sigma\left(X_{t}\right) d B_{s}+\int_{0}^{t} d s .
$$

Lấy kỳ vọng hai vế ta được

$\mathbb{E}\left\{f_{1}\left(X_{t}\right)\right\}=t$.

Như vậy (3.2) đã được chứng minh.

Khi đó, với mọi $\varepsilon>0, \exists M>0$ sao cho $\forall|x|>M$ thì

$$
\left|1-\frac{f_{1}(x)}{x} \times \frac{1}{T(\lambda)}\right|<\varepsilon .
$$

Phân tích $\Omega=\left\{\left|X_{t}\right| \leq M\right\} \cup\left\{\left|X_{t}\right|>M\right\}$, kết hợp các tính chất (3.2) và (3.3) ta có các ước lượng như sau

$$
\begin{aligned}
\left|\mathbb{E}\left\{\frac{X_{t}}{t}\right\}-\frac{1}{T(\lambda)}\right| & =\left|\mathbb{E}\left\{\frac{X_{t}}{t}-\frac{f_{1}\left(X_{t}\right)}{t} \frac{1}{T(\lambda)}\right\}\right| \\
\leq \mathbb{E}\left\{\mid 1-\frac{f_{1}\left(X_{t}\right)}{X_{t}}\right. & \left.\times \frac{1}{T(\lambda)} \mid \frac{\left|X_{t}\right|}{t} 1_{\left\{\left|X_{t}\right|>M\right\}}\right\} \\
& +\frac{1}{t} \mathbb{E}\left\{\mid X_{t}\right. \\
& \left.-f_{1}\left(X_{t}\right) \frac{1}{T(\lambda)} \mid 1_{\left\{\left|X_{t}\right| \leq M\right\}}\right\} .
\end{aligned}
$$

Số hạng thứ nhất có chặn trên là

$\varepsilon \mathbb{E}\left\{\frac{\left|X_{t}\right|}{t} 1_{\left\{\left|X_{t}\right|>M\right\}}\right\} \leq \varepsilon \mathbb{E}\left\{\frac{\left|X_{t}\right|}{t}\right\} \leq \varepsilon \cdot \sqrt{\mathbb{E}\left\{\left(\frac{X_{t}}{t}\right)^{2}\right\}}$

và số hạng còn lại

$\frac{1}{t} \mathbb{E}\left\{\left|X_{t}-f_{1}\left(X_{t}\right) \frac{1}{T(\lambda)}\right| 1_{\left\{\left|X_{t}\right| \leq M\right\}}\right\} \stackrel{t \rightarrow+\infty}{\longrightarrow} 0$. 
Như vậy mệnh đề 3.1 sẽ được chứng minh nếu như $\mathbb{E}\left(X_{t}^{2} / t^{2}\right)$ bị chặn. Điều đó sẽ được chỉ ra trong mệnh đề kế tiếp.

Mệnh đề 3.2 Với quá trình khuếch tán đã cho

$$
\lim _{t \rightarrow+\infty} \mathbb{E}\left\{\left(\frac{X_{t}}{t}\right)^{2}\right\}=\left(\frac{\lambda \beta}{\alpha}\right)^{2}=\frac{1}{T^{2}(\lambda)} .
$$

Chứng minh. Xét hàm số không âm $f_{2}$, xác định trên $\mathbb{R}$, sao cho

$$
\left\{\begin{array}{c}
L f_{2}(x)=f_{1}(x) \\
f_{2}(0)=0
\end{array} .\right.
$$

Áp dụng Bổ đề 2.2 thì các nghiệm của phương trình đã cho là

$$
\begin{aligned}
& f_{2}(x) \\
& =\left\{\begin{array}{cc}
\int_{0}^{x} \frac{1}{e^{2 \lambda v} b(v)} \int_{-\infty}^{v} 2 e^{2 \lambda u} a(u) f_{1}(u) d u d v, & x \geq 0 \\
-\int_{x}^{0} \frac{1}{e^{2 \lambda v} b(v)} \int_{-\infty}^{v} 2 e^{2 \lambda u} a(u) f_{1}(u) d u d v, & x<0
\end{array}\right.
\end{aligned}
$$

Tương tự như trên thì giới hạn sau cũng đúng

$$
\lim _{x \rightarrow \infty} \frac{f_{2}(x)}{x^{2}}=\frac{1}{2}\left(\frac{\alpha}{\lambda \beta}\right)^{2}=\frac{T^{2}(\lambda)}{2} .
$$

và hơn nữa

$$
\mathbb{E}\left\{f_{2}\left(X_{t}\right)\right\}=\frac{t^{2}}{2} \quad, \forall t \geq 0 .
$$

Khi đó, với mọi $\varepsilon>0, \exists M>0$ sao cho $\forall|x|>$ $M$ thì

$$
\left|\frac{x^{2}}{f_{2}(x)}-\frac{2}{T^{2}(\lambda)}\right|<\varepsilon
$$

Phân tích $\Omega=\left\{\left|X_{t}\right| \leq M\right\} \cup\left\{\left|X_{t}\right|>M\right\}$, kết hợp các tính chất (3.4) và (3.5) ta có các ước lượng như sau

$$
\begin{aligned}
&\left|\mathbb{E}\left\{\left(\frac{X_{t}}{t}\right)^{2}\right\}-\frac{1}{T^{2}(\lambda)}\right|\left|\mathbb{E}\left\{\left(\frac{X_{t}}{t}\right)^{2}-\frac{f_{2}\left(X_{t}\right)}{t^{2}} \frac{2}{T^{2}(\lambda)}\right\}\right| \\
& \leq \mathbb{E}\left\{\left|\frac{X_{t}^{2}}{f_{2}\left(X_{t}\right)}-\frac{2}{T^{2}(\lambda)}\right| \frac{f_{2}\left(X_{t}\right)}{t^{2}} 1_{\left\{\left|X_{t}\right|>M\right\}}\right\} \\
&+\frac{1}{t^{2}} \mathbb{E}\left\{\mid X_{t}^{2}\right. \\
&\left.-f_{2}\left(X_{t}\right) 2 T^{2}(\lambda) \mid 1_{\left\{\left|X_{t}\right| \leq M\right\}}\right\}
\end{aligned}
$$

Số hạng thứ nhất có chặn trên là $\varepsilon \mathbb{E}\left\{\frac{f_{2}\left(X_{t}\right)}{t^{2}} 1_{\left\{\left|X_{t}\right|>M\right\}}\right\} \leq \varepsilon \mathbb{E}\left\{\frac{f_{2}\left(X_{t}\right)}{t^{2}}\right\}=\frac{\varepsilon}{2}<\varepsilon$

và số hạng còn lại

$$
\frac{1}{t^{2}} \mathbb{E}\left\{\left|X_{t}^{2}-f_{2}\left(X_{t}\right) \frac{2}{T^{2}(\lambda)}\right| 1_{\left\{\left|X_{t}\right| \leq M\right\}}\right\} \stackrel{t \rightarrow+\infty}{\longrightarrow} 0 \text {. }
$$

Như vậy mệnh đề 3.2 đã được chứng minh.

\section{KẾT LUẬN}

Bài báo đã chứng minh luật số lớn cho quá trình khuếch tán trên không gian trạng thái $\mathbb{R}$ thông qua việc sử dụng phương pháp moment. Ngoài ra, điểm mấu chốt trong bài toán này là có thể giải được phương trình Poisson tương ứng với toán tử cực vi $L$. Phương pháp này được kỳ vọng có thể được áp dụng cho các bài toán khác có liên quan.

\section{TÀI LIỆU THAM KHẢO}

Billingsley, P. (1995). Probability and measure, Third Edition. Wiley. New York, 593 pages.

Depauw, J., \& Derrien, J. M. (2009). Variance limite d'une marche aléatoire réversible en milieu aléatoire sur Z. Comptes Rendus Mathematique. 347(7-8): 401-406.

Lam, H. C. (2014). A quenched central limit theorem for reversible random walks in a random environment on Z. Journal of Applied Probability, 51(4), 1051-1064.

Lâm Hoàng Chương, Dương Thị Bé Ba, Lê Hoài Nhân \& Trần Thị Thiện (2019). Định lý giới hạn trung tâm cho quá trình khuếch tán trong không gian một chiều. Tạp chi Khoa học Truờng Đại hoc Cần Tho: 55(6A): 37-41.

Papanicolaou, G. C., \& Varadhan, S. R. S. (1982). Diffusions with random coefficients. In Kallianpur, G., Krishnaiah, P. R. and Ghosh, J.K. (Eds.), Statistics and Probability: Essays in Honor of C. R. Rao, North-Holland, Amsterdam, pp. 547-552. 